\title{
Models of Inflammation: Carrageenan- or Complete Freund's Adjuvant-Induced Edema and Hypersensitivity in the Rat
}

\author{
Jill C. Fehrenbacher ${ }^{A}$, Michael R. Vasko ${ }^{A}$, and Djane B. Duarte ${ }^{A}$ \\ ADepartment of Pharmacology and Toxicology, Indiana University School of Medicine, \\ Indianapolis, IN 46202
}

\begin{abstract}
Animal models of inflammation are used to assess the production of inflammatory mediators at sites of inflammation, the anti-inflammatory properties of agents such as nonsteroidal antiinflammatory drugs (NSAIDs), and the efficacy of putative analgesic compounds to reverse cutaneous hypersensitivity. This protocol details methods to elicit and measure carrageenan- and complete Freund's adjuvant-induced cutaneous inflammation. Due to possible differences between the dorsal root sensory system and the trigeminal sensory system, injections of either the footpad or vibrissal pad are described. In this manner, cutaneous inflammation can be assessed in tissue innervated by the lumbar dorsal root ganglion neurons (footpad) and by the trigeminal ganglion neurons (vibrissal pad).
\end{abstract}

\section{Keywords}

inflammation; carrageenan; Complete Freund's Adjuvant; edema; nociception

\section{BASIC PROTOCOL}

\section{Introduction}

A solution of carrageenan in saline injected subcutaneously in rats induces an acute swelling that becomes maximal 3-5 hours after the injection and subsides by 24 hours (Winter et al., 1962; Otterness and Moore, 1988), whereas a subcutaneous injection of complete Freund's adjuvant in saline induces a more prolonged swelling that becomes maximal at $24 \mathrm{hrs}$ and persists for at least 7 days (Iadarola et al., 1988; Stein et al., 1988). The inflammation produced in these models can be used to assess the production of inflammatory mediators at sites of inflammation, the anti-inflammatory properties of agents such as nonsteroidal antiinflammatory drugs (NSAIDs), and the efficacy of putative analgesic compounds to reverse cutaneous hypersensitivity. This protocol details methods to elicit and measure carrageenanand complete Freund's adjuvant-induced inflammation in the footpad or vibrissal pad, which can manifest as edema and hypersensitivity.

Corresponding author: Jill C. Fehrenbacher, Department of Pharmacology and Toxicology, Indiana University, School of Medicine, 635 Barnhill Drive, MSA419, Indianapolis, Indiana 46202, Telephone: 317.274.8360, Fax: 317.274.7714, jfehrenb@iupui.edu. 
Due to possible differences between the dorsal root sensory system and the trigeminal sensory system (Bereiter et al., 2009), cutaneous injections of either the footpad or vibrissal pad are described. In this manner, cutaneous inflammation can be assessed in tissue innervated by the lumbar dorsal root ganglion neurons (footpad) and by the trigeminal ganglion neurons (vibrissal pad). This protocol focuses on the induction of inflammation in rats, however it can easily be adapted for mice $>20 \mathrm{~g}$ by using smaller injection volumes (i.e., $50 \mu \mathrm{l}$ for vibrissal pad and 25-50 $\mu \mathrm{l}$ for the footpad, Cunha et al., 2004; Schreiber $e t$ al., 2008; Poh et al., 2009) and a $3 \mathrm{~mm}$ biopsy punch.

NOTE: All protocols using live animals must first be reviewed and approved by an Institutional Animal Care and Use Committee (IACUC) or must conform to governmental regulations regarding the care and use of laboratory animals.

\section{Materials list}

Male or female rats weighing $>100 \mathrm{~g}$ upon arrival

$0.5-2.0 \%$ (w/v) carrageenan solution (Type IV Lambda, Sigma)

Complete Freund's adjuvant (1 mg/ml of Mycobacterium tuberculosis, heat killed and dried; Sigma)

$0.9 \%(\mathrm{w} / \mathrm{v})$ saline, sterile

Inhalational anesthesia and/or animal restrainer

Scales, accurate to 0.1 and $0.0001 \mathrm{~g}$

Betadine

Ethanol $(70 \%, v / v)$

23-G, $1 / 2$-in needle

3-ml glass syringe

27-G, $1 / 2$-in needle

1-ml syringes

$6 \mathrm{~mm}$ biopsy punch

\section{Induction of edema}

1 Allow animals to acclimate for 1 week in solid-bottom cages. It is preferable to use wood shavings in the bottom of the cages because they provide a softer surface for the irritated footpads than pellets of compressed grain or paper products. Animals should be acclimated under standard lighting and temperature conditions with food and water ad libitum to eliminate the effects of stress. Weigh the rats using a scale accurate to $0.1 \mathrm{~g}$.

2 Prepare syringes:

Footpad injections: 
Carrageenan: for each animal, load a sterile $1 \mathrm{cc}$ syringe with a $27-\mathrm{G}, 1 / 2-$ in needle attached with $100 \mu \mathrm{l}$ of the carrageenan solution.

CFA: for each animal, load a sterile 1cc syringe (needle unattached) with $\sim 250$ $\mu \mathrm{l}$ of CFA emulsion. Wipe the tip of the syringe and attach a $27-\mathrm{G}, 1 / 2$-in needle. Discard the excess CFA through the needle until only $100 \mu \mathrm{l}$ remains in the syringe.

Vibrissal pad injections:

Carrageenan: for each animal, load a sterile 1cc syringe with a 27-G, -in needle attached with $100 \mu \mathrm{l}$ of carrageenan.

CFA: for each animal, load a sterile 1cc syringe (needle unattached) with $\sim 250$ $\mu \mathrm{l}$ of CFA emulsion. Wipe the tip of the syringe and attach a $27-\mathrm{G}, 1 / 2$-in needle. Discard the excess CFA through the needle until only $100 \mu \mathrm{l}$ remains in the syringe.

3 Injection of the footpad:

Place the rat in a temporary restraint or place the rat in a clear plastic box with inhalational anesthesia until the rat is unconscious, being careful not to kill the animal. Typically, a concentration of 5\% isoflurane (v/v) is sufficient to induce anesthesia. Once animals have lost their righting reflex, they will remain anesthetized for $\sim 15-20$ seconds, which is adequate time to clean and inject the paw. Clean the plantar surface on the footpad with betadine and $70 \%$ ethanol. Insert the needle, bevel up, into the metatarsal region of the hindpaw (just proximal to the distal calluses) almost parallel to the footpad (Figure 1A). Move the needle 3-4 mm proximally towards the tarsal region and deliver the solution (100 $\mu$ carrageenan, CFA, or saline). Place your thumb lightly on the foot pad while withdrawing the needle and maintain pressure for 10 seconds. Return the rat to its cage after injection. Repeat the injection for each animal. Figure 2 illustrates the degree of inflammation in the foot pad 24 hours after the injection of saline or CFA with paw thickness measured using a micrometer.

4 Injection of the vibrissal pad:

Place the rat in a clear plastic box with inhalational anesthesia until the rat is unconscious, being careful not to kill the animal. Clean the vibrissal pad with betadine and $70 \%$ ethanol. Insert the needle, bevel facing away from the rat just posterior to the nose in the $2^{\text {nd }}$ row of vibrissae. Move the needle $\sim 3 \mathrm{~mm}$ towards the eye and deliver the solution (100 $\mu \mathrm{l}$ carrageenan, CFA, or saline). Place your thumb lightly on the vibrissal pad while withdrawing the needle and maintain pressure for 10 seconds. Return the rat to its cage after injection. Repeat the injection for each animal.

Measurement of edema-The mass of a punch tissue biopsy will be utilized to determine the degree of edema within the footpad or vibrissal pad. Sacrifice the rat at the desired time following saline, carrageenan, or CFA-injection. 
5 For the footpad, use a $6 \mathrm{~mm}$ biopsy punch to remove a section of tissue. Use the calluses as anatomical markers and punch between them all of the way to the tendons to achieve consistency between animals (Figure 1B). Weigh the tissue on a scale accurate to $0.0001 \mathrm{~g}$ and calculate the difference between the salineand irritant-injected punches.

6 For the vibrissal pad, make a midline incision from between the ears to the nose. Dissect away the skin so that the inside of the vibrissal pad is accessible. Use a $6 \mathrm{~mm}$ biopsy punch to remove a section of the vibrissal pad from the inside of the skin outwards. Use the vibrissae as anatomical markers and make a punch centered on the third row of vibrissae to achieve consistency between animals. Weigh the tissue on a scale accurate to $0.0001 \mathrm{~g}$ and calculate the difference in mass between the saline- and irritant-injected punches.

\section{REAGENTS AND SOLUTIONS}

Carrageenan: $0.5 \%-2.0 \%(\mathrm{w} / \mathrm{v})$ carrageenan solution

Pour $500 \mathrm{ml}$ of sterile $0.9 \%$ saline into a 1-liter beaker and add a stir bar. Slowly add $2.5 \mathrm{~g}(0.5 \%)-10.0 \mathrm{~g}(2.0 \%)$ carrageenan powder (Type IV Lambda, Sigma) to the beaker. Heat the solution to $90^{\circ} \mathrm{C}$ with stirring, but do not allow the solution to boil. Heating the mixture helps to dissolve the carrageenan; it should take less than an hour to dissolve all of the powder. Pour the solution into a clean 1-liter glass bottle equipped with a cap and sterilize by autoclaving. Cool the solution to room temperature and aliquot into sterile, 100-ml capped bottles. This solution is stable for at least a week when stored at $4^{\circ} \mathrm{C}$ following preparation.

Complete Freund's adjuvant: $0.5 \mathrm{mg} / \mathrm{ml}$ mycobacterium emulsion

Vortex the bottle of CFA (Sigma). This step is very important because it eliminates the settling of the Mycobacteria during storage. Add $0.5 \mathrm{ml}$ of CFA to $0.5 \mathrm{ml}$ of sterile $0.9 \%$ saline. Using a $3 \mathrm{cc}$ glass syringe with a $23-\mathrm{G}, 3 / 4-$ in needle attached, mix the solution approximately eight times to create a homogeneous emulsion by drawing the solution in and out of the syringe. This solution should be prepared fresh each time injections will be made.

\section{COMMENTARY}

\section{Background Information}

The subcutaneous injection of either carrageenan or complete Freund's adjuvant produces local inflammation, designated by the 5 cardinal signs: hypersensitivity, redness, swelling, heat, and loss of function (Winter et al., 1962; Stein et al., 1988). The models described in this protocol use carrageenan as an irritant to induce a transient inflammation or complete Freund's adjuvant to induce a more persistent inflammation. Edema (swelling), hypersensitivity to a noxious stimulus (hyperalgesia), or sensitivity to a nonnoxious stimulus (allodynia) can be used to assess inflammation in an animal model, thus providing multiple methods to monitor inflammatory responses and the reversal or prevention of these responses with anti-inflammatory compounds, such as NSAIDs. Various methods have been published to monitor edema; some measure the size of the paw via volume displacement 
(plethysometry), while others measure the width, diameter or circumference of the paw before and after inflammation (Singh and Ghosh, 1968; Iadarola et al., 1988). Here, a simple procedure using biopsy punches is presented (representative values for carrageenan- and CFA-induced increases in punch mass are presented in Table 1). The behaviors used to determine hypersensitivity include unlearned behaviors such as flinching, vocalization, and grooming (D'Amour and Smith, 1941; Woolfe and MacDonald, 1944; Randall and Selitto, 1957) or segmental withdrawal reflexes in response to thermal or mechanical stimulation (Hargreaves et al., 1988; Chaplan et al., 1994). Using models of inflammation induced by either carrageenan or CFA, researchers have demonstrated that the NSAID, indomethacin, reduces paw swelling in a dose-dependent manner by at least $60 \%(1-9 \mathrm{mg} / \mathrm{kg}$, ip; Winter $e t$ al., 1962; Winter et al., 1968; Mirshafiey et al., 2005) and attenuates hyperalgesia and allodynia (2-4 mg/kg, ip; Hargreaves et al., 1988; Tall et al., 2004; Zhang et al., 2004). Interestingly, these components of inflammation (edema and hypersensitivity) do not necessarily go hand in hand. For example, both glutamate and calcium channel antagonists can reverse inflammatory hypersensitivity without ameliorating the edema (Walker et al., 2001; Brittain et al., 2011).

\section{Critical Parameters}

Rats must be acclimated at least 1 week prior to initiation of the experiment to eliminate the effects of stress. The degree of swelling and hypersensitivity depends on the carrageenan and CFA preparations and the injections. When new lots of the irritants are used, they should be titrated to define the most consistent induction of edema and hypersensitivity formation and to compare the effectiveness with that of the previous lot(s). Although higher concentrations of these irritants can produce increased swelling, the maximal hypersensitivity and edema responses may prove to be too difficult to inhibit. Most animal care review panels limit the acceptable concentration of CFA to $0.5 \mathrm{mg}$ Mycobacterium $/ \mathrm{ml}$ and will not approve the use of emulsion volumes larger than $100 \mu$ into the footpad to minimize the occurrence of unwanted skin ulceration and tissue sloughing (Jackson and Fox, 1995). Therefore, it is useful to determine the best therapeutic window of hypersensitivity and/or edema to use for assays to determine inhibitory actions of anti-inflammatory and/or analgesic drugs.

\section{Troubleshooting}

Inconsistencies in the degree of inflammation induced by CFA can be due to settling of the Mycobacterial cellular debris, so it is recommended that the investigators vortex and visually inspect the vial of CFA prior to pipetting the solution. Another potential source of inconsistency is that the thick emulsion of CFA leaks from the puncture wound made by the needle during injection. Investigators are thus advised to maintain pressure on the paw until an initial blood clot forms. Obtaining reproducible punch biopsies takes practice; punching all of the way to the tendons in the footpad biopsies is recommended to achieve consistency between animals. 


\section{Anticipated Results}

An increase in the weight of the punch biopsy secondary to injection of carrageenan or CFA is expected. It is unethical to induce inflammation in both footpads, so the effects of antiinflammatory drugs should be investigated between groups of animals. Typically, the salineinjected footpad biopsy weighs $\sim 25 \mathrm{mg}$, whereas the vibrissal pad biopsy weighs $\sim 60 \mathrm{mg}$. The paw injected with carrageenan or CFA shows a 95-120 mg increase and the vibrissal pad injected with the inflammatory agents shows an increase of 40-50 mg (see Table 1). Figure 3 demonstrates the effect of the NSAID, ketorolac, on thermal hypersensitivity and edema induced by carrageenan injection into the footpad. The withdrawal latency to a thermal stimulus is illustrated in panel A for saline and carrageenan injected animals $(n=3)$. Administration of ketorolac (10mg/kg, i.p.; indicated by the dark fill) 30 minutes prior to carrageenan injection completely reverses the decrease in paw withdrawal latency induced by inflammation ( $\mathrm{p}<0.05$ determined by a t-test between vehicle- and ketorolac-treated animals). In contrast, the edema induced by carrageenan injection (panel B; measured by the mass of a paw punch biopsy) is only partially attenuated by ketorolac administration (indicated by the dark fill; $\mathrm{p}<0.05$ determined by a t-test between vehicle- and ketorolactreated animals). Ketorolac did not alter the paw withdrawal latency or the paw punch biopsy mass in saline injected animals.

\section{Time Considerations}

The carrageenan solution is generally prepared in advance and stored. The CFA emulsion takes $\sim 10$ minutes to prepare. The animal procedure for the induction of inflammation takes roughly 10 minutes per animal. The in vivo incubation time is dependent on which model is used: $\sim 3$ hours for carrageenan and 6-60 hours for CFA. Recovery of biopsy punches and punch mass determination requires $\sim 5$ minutes per animal.

\section{Acknowledgments}

Funding: NIDCR DE018796 to JCF and NINDS NS069915 to MRV

\section{Literature cited}

Bereiter, DA.; Hargreaves, KM.; Hu, JW. Trigeminal mechanisms of nociception: peripheral and brain stem organization. In: Bushnell, MC.; Basbaum, AI., editors. Handbook of the Senses, vol 5, Science of Pain. Elsevier; San Diego: 2009. p. 435-460.

Brittain J, Duarte D, Wilson S, Wang Y, Zhu W, Ballard C, Khanna M, Brustovetsky T, Schmutzler B, Xiong W, Ripsch M, Ashpole N, Hudmon A, Meroueh S, Hingtgen C, Brustovesky N, Jim X, Vasko M, Fehrenbacher J, Hurley J, White F, Khanna R. Suppression of inflammatory and neuropathic pain by uncoupling CRMP-2 from the presynaptic $\mathrm{Ca} 2+$ channel complex. Nature Medicine. 2011 Article in Press.

Chaplan SR, Bach FW, Pogrel JW, Chung JM, Yaksh TL. Quantitative assessment of tactile allodynia in the rat paw. J Neurosci Methods. 1994; 53:55-63. [PubMed: 7990513]

Cunha TM, Verri WA Jr, Vivancos GG, Moreira IF, Reis S, Parada CA, Cunha FQ, Ferreira SH. An electronic pressure-meter nociception paw test for mice. Braz J Med Biol Res. 2004; 37:401-7. [PubMed: 15060710]

D'Amour F, Smith D. A method for determining loss of pain sensation. J Pharmacol Exp Ther. 1941; 72:74-79. 
Hargreaves K, Dubner R, Brown F, Flores C, Joris J. A new and sensitive method for measuring thermal nociception in cutaneous hyperalgesia. Pain. 1988; 32:77-88. [PubMed: 3340425]

Iadarola MJ, Brady LS, Draisci G, Dubner R. Enhancement of dynorphin gene expression in spinal cord following experimental inflammation: stimulus specificity, behavioral parameters and opioid receptor binding. Pain. 1988; 35:313-26. [PubMed: 2906426]

Jackson LR, Fox JG. Institutional Policies and Guidelines on Adjuvants and Antibody Production. ILAR J. 1995; 37:141-152. [PubMed: 11528034]

Mirshafiey A, Cuzzocrea S, Rehm B, Mazzon E, Saadat F, Sotoude M. Treatment of experimental arthritis with M2000, a novel designed non-steroidal anti-inflammatory drug. Scand J Immunol. 2005; 61:435-41. [PubMed: 15882435]

Otterness IG, Moore PF. Carrageenan foot edema test. Methods Enzymol. 1988; 162:320-7. [PubMed: 3226312]

Poh KW, Lutfun N, Manikandan J, Ong WY, Yeo JF. Global gene expression analysis in the mouse brainstem after hyperalgesia induced by facial carrageenan injection--evidence for a form of neurovascular coupling? Pain. 2009; 142:133-41. [PubMed: 19167818]

Randall LO, Selitto JJ. A method for measurement of analgesic activity on inflamed tissue. Arch Int Pharmacodyn Ther. 1957; 111:409-19. [PubMed: 13471093]

Schreiber KL, Beitz AJ, Wilcox GL. Activation of spinal microglia in a murine model of peripheral inflammation-induced, long-lasting contralateral allodynia. Neurosci Lett. 2008; 440:63-7. [PubMed: 18541374]

Singh H, Ghosh MN. Modified plethysmometer for measuring foot volume of unanesthetized rats. J Pharm Pharmacol. 1968; 20:316-7. [PubMed: 4384613]

Stein C, Millan MJ, Herz A. Unilateral inflammation of the hindpaw in rats as a model of prolonged noxious stimulation: alterations in behavior and nociceptive thresholds. Pharmacol Biochem Behav. 1988; 31:445-51. [PubMed: 3244721]

Tall JM, Seeram NP, Zhao C, Nair MG, Meyer RA, Raja SN. Tart cherry anthocyanins suppress inflammation-induced pain behavior in rat. Behav Brain Res. 2004; 153:181-8. [PubMed: 15219719]

Walker K, Bowes M, Panesar M, Davis A, Gentry C, Kesingland A, Gasparini F, Spooren W, Stoehr N, Pagano A, Flor PJ, Vranesic I, Lingenhoehl K, Johnson EC, Varney M, Urban L, Kuhn R. Metabotropic glutamate receptor subtype 5 (mGlu5) and nociceptive function. I. Selective blockade of mGlu5 receptors in models of acute, persistent and chronic pain. Neuropharmacology. 2001; 40:1-9. [PubMed: 11077065]

Winter CA, Risley EA, Nuss GW. Carrageenin-induced edema in hind paw of the rat as an assay for antiiflammatory drugs. Proc Soc Exp Biol Med. 1962; 111:544-7. [PubMed: 14001233]

Winter CA, Risley EA, Silber RH. Antiinflammatory activity of indomethacin and plasma corticosterone in rats. J Pharmacol Exp Ther. 1968; 162:196-201. [PubMed: 5656598]

Woolfe G, MacDonald A. The evaluation of the analgesic action of pethidine hydrochloride (Demerol). J Pharmacol Exp Ther. 1944; 80:300-307.

Zhang RX, Lao L, Wang X, Ren K, Berman BB. Electroacupuncture combined with indomethacin enhances antihyperalgesia in inflammatory rats. Pharmacol Biochem Behav. 2004; 78:793-7. [PubMed: 15301937] 

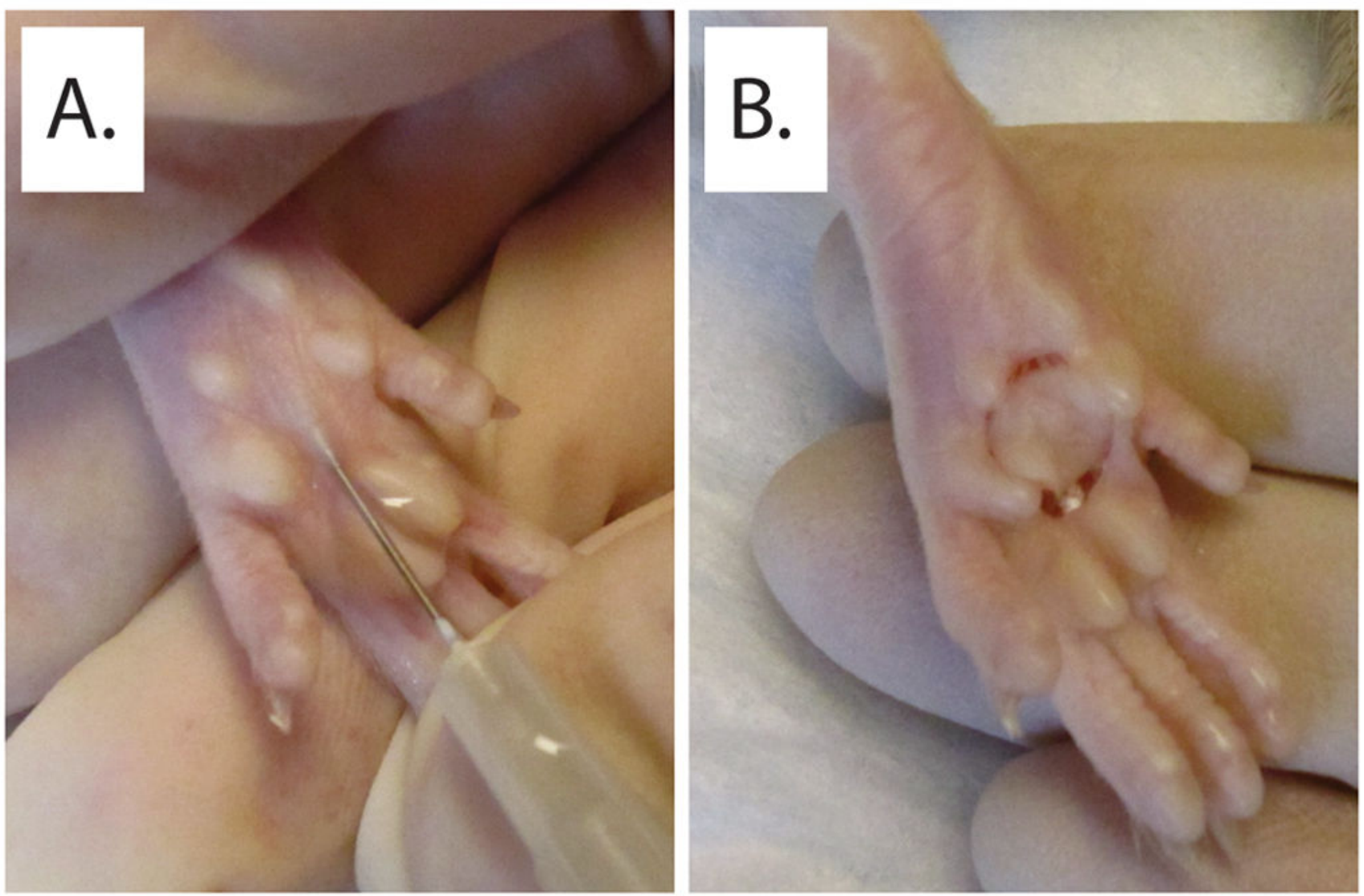

Figure 1.

(A). Site of injection of proinflammatory substances into the footpad of the rat. (B). Optimal area for paw punch biopsies to obtain consistent results. 


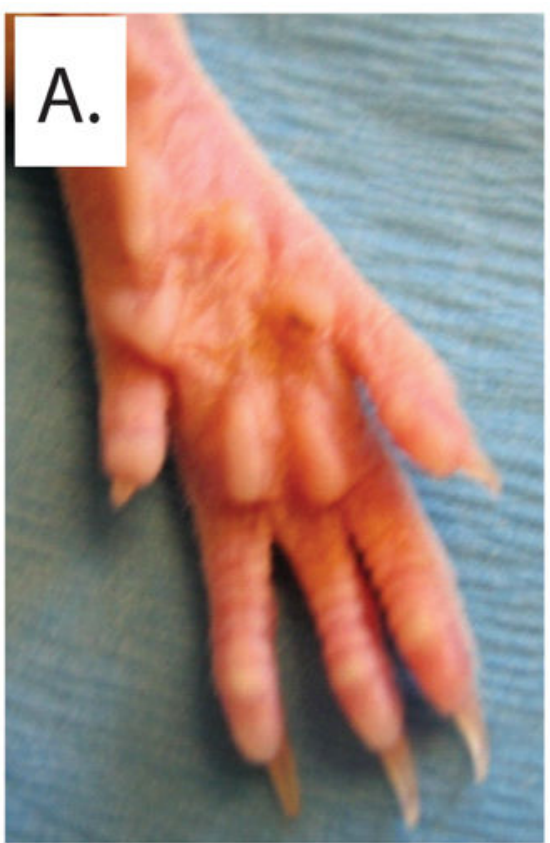

Saline

Paw thickness, mm: (24-hr postinjection) $\quad 2.43 \pm 0.09 \mathrm{~mm}$

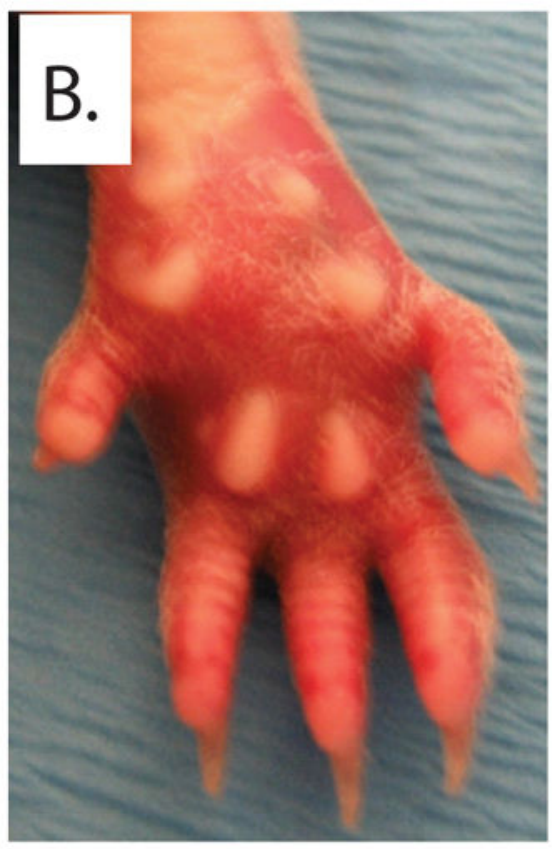

CFA

Figure 2.

(A). Photograph of a footpad from an animal $24 \mathrm{hrs}$ after injection with $100 \mu \mathrm{l}$ saline. (B). Photograph of a footpad from an animal $24 \mathrm{hrs}$ after injection with $100 \mu \mathrm{l} \mathrm{CFA}(0.5 \mathrm{mg} / \mathrm{ml})$. 
A.

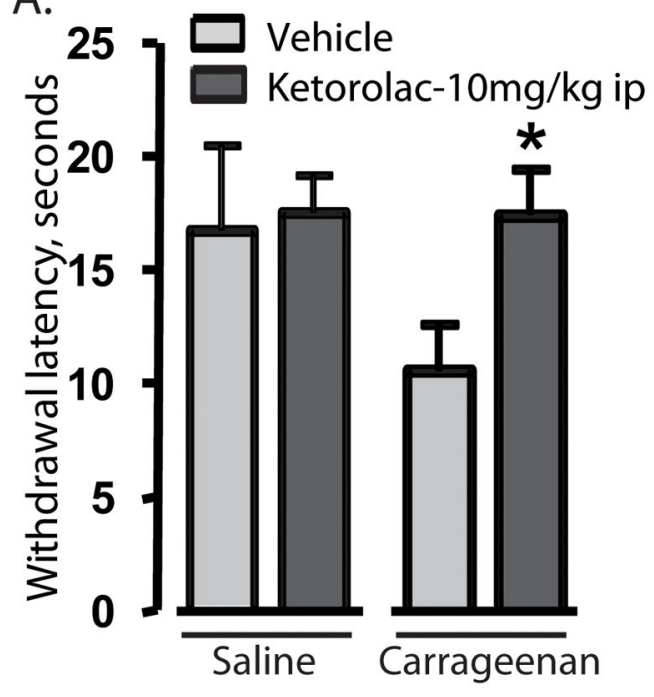

B.

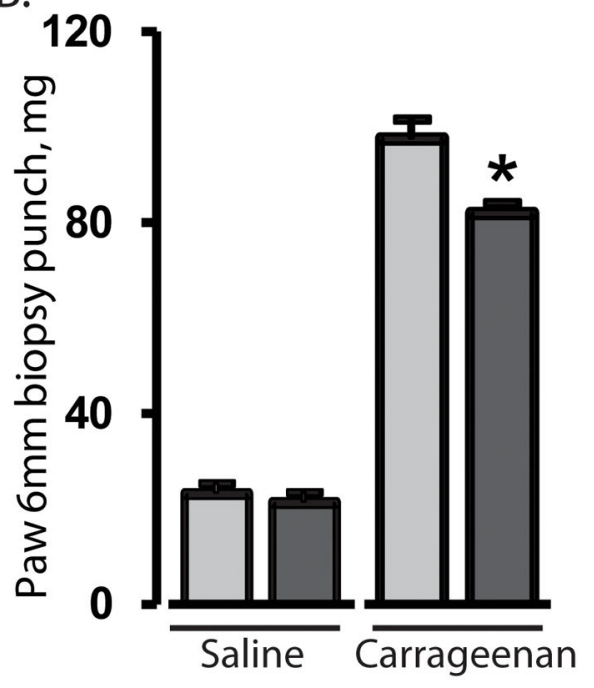

Figure 3.

Effects of ketorolac on carrageenan-induced thermal hyperalgesia and edema. (A) Thermal hypersensitivity in animals injected with saline (100 $\mu \mathrm{l}$; animal tested $3 \mathrm{hrs}$ after injection) or carrageenan ( $100 \mu \mathrm{l}$ of $0.5 \%$; animal tested $3 \mathrm{hrs}$ after injection). Thermal hypersensitivity is expressed as the paw withdrawal latencies (seconds) to a thermal stimulation using the Hargreaves apparatus. Ketorolac ( $10 \mathrm{mg} / \mathrm{kg}$, ip; dark fill) administered 30 minutes prior to carrageenan injection completely reversed the inflammation-induced thermal hypersensitivity $(\mathrm{p}<0.05$ determined by a t-test between vehicle- and ketorolac-treated animals, $n=3$ /group). (B) Edema induced by saline or carrageenan injection. The edema is expressed as the wet weight (mg) of paw punch biopsy mass. Ketorolac $(10 \mathrm{mg} / \mathrm{kg}$, ip; dark fill) administered 30 minutes prior to carrageenan injection partially reversed the inflammation-induced edema $(\mathrm{p}<0.05$ determined by a t-test between vehicle- and ketorolactreated animals, $n=3$ /group). 


\section{Table 1}

Carrageenan- and CFA-induced edema in the footpad or vibrissal pad.

\begin{tabular}{|l|l|l|l|}
\hline & Saline & Carrageenan $(\mathbf{0 . 5 \%})$ & CFA $(\mathbf{0 . 5 m g} / \mathbf{m l})$ \\
\hline Footpad punch & $23.7 \pm 1.4 \mathrm{mg}$ & $93.5 \pm 1.2 \mathrm{mg}$ & $116.8 \pm 8.2 \mathrm{mg}$ \\
\hline Vibrissal pad punch & $61.2 \pm 2.2 \mathrm{mg}$ & $101.1 \pm 4.0 \mathrm{mg}$ & $111.8 \pm 11.5 \mathrm{mg}$ \\
\hline
\end{tabular}

Carrageenan-injected animals were sacrificed 3 hours after injection, whereas CFA-injected animals were sacrificed 24 hours after injection and masses of 6-mm biopsy punches were taken from the respective sites. Values presented are mean \pm standard error of the mean in milligrams for each group, $\mathrm{n}=3$. 